\title{
ALDH2 rs671 polymorphisms and the risk of cerebral microbleeds in Chinese elderly: the Taizhou Imaging Study
}

\author{
Zhen Zhu ${ }^{1,2 \#}$, Yanfeng Jiang ${ }^{2,3 \#}$, Mei Cui ${ }^{4}$, Yingzhe Wang ${ }^{4}$, Shuyuan $\mathrm{Li}^{5}$, Kelin $\mathrm{Xu}^{2,6}$, Kexun $\mathrm{Zhang}^{1,2}$, \\ Chengkai Zhu ${ }^{2,7}$, Wanghong $\mathrm{Xu}^{1}$, Li Jin ${ }^{2,3,7}$, Weimin $\mathrm{Ye}^{2,8}$, Chen Suo ${ }^{1,2}$, Xingdong Chen ${ }^{2,3}$ \\ ${ }^{1}$ Department of Epidemiology, School of Public Health, and the Key Laboratory of Public Health Safety of Ministry of Education, Fudan University, \\ Shanghai 200438, China; ${ }^{2}$ State Key Laboratory of Genetic Engineering, Human Phenome Institute, Fudan University, Shanghai 200438, China; \\ ${ }^{3}$ Fudan University Taizhou Institute of Health Sciences, Taizhou 225312, China; ${ }^{4}$ Department of Neurology, Huashan Hospital, Fudan University, \\ Shanghai 200030, China; International Peace Maternity and Child Health Hospital, School of Medicine, Shanghai Jiao Tong University, Shanghai \\ 200030, China; 'School of Data Science and Institute for Big Data, and the Collaborative Innovation Center for Genetics and Development, Fudan \\ University, Shanghai 200433, China; ${ }^{7}$ School of Life Sciences, Fudan University, Shanghai 200438, China; ${ }^{8}$ Department of Medical Epidemiology \\ and Biostatistics, Karolinska Instituted, Stockholm, Sweden \\ Contributions: (I) Conception and design: X Chen, C Suo, Y Jiang; (II) Administrative support: X Chen, C Suo, Y Jiang; (III) Provision of study \\ materials or patients: L Jin, X Chen; (IV) Collection and assembly of data: Z Zhu, Y Jiang, K Xu, K Zhang; (V) Data analysis and interpretation: Z \\ Zhu, Y Jiang; (VI) Manuscript writing: All authors; (VII) Final approval of manuscript: All authors. \\ \#These authors contributed equally to this work. \\ Correspondence to: Xingdong Chen, PhD. School of Life Sciences, Fudan University, Songhu Road 2005, Shanghai 200438, China. \\ Email: xingdongchen@fudan.edu.cn; Chen Suo, PhD. School of Public Health, Fudan University, Dong'An Road 138, Shanghai 200032, China. \\ Email: suochen@fudan.edu.cn.
}

Background: Cerebral microbleeds (CMBs) are more prevalent in Asian populations, and have been associated with increased risk of stroke, dementia and mortality. So far, risk factors for CMBs other than hypertension were merely known. Previous studies have shown that polymorphisms at aldehyde dehydrogenase $2(A L D H 2)$ gene were independently associated with the risk of stroke. Its role in CMBs, however, remains unclear. This study aimed to evaluate the associations of $A L D H 2$ gene polymorphisms with CMBs in Chinese elderly.

Methods: Using bio-specimen and data collected at baseline survey of the population-based Taizhou Imaging Study (TIS) (phase I), we genotyped the single nucleotide polymorphisms (SNPs) at $A L D H 2$ among 549 individuals aged 55-65 years, and rs671 was used as surrogate marker of ALDH2. CMBs were detected on brain magnetic resonance imaging (MRI), and further categorized as strictly lobar or as deep/mixed. Logistic regression models were used to evaluate the associations of the variants at $A L D H 2$ and CMBs.

Results: CMBs were present in 103 individuals $(18.8 \%)$. Forty-one point three percent participants were with $A L D H 2 * 2$ allele and $5.1 \%$ had $A L D H 2 * 2 /{ }^{*} 2$ genotype. Subjects with $A L D H 2 * 1$ allele were more likely to be drinker, have hypertension or $\mathrm{CMBs}$ than those with *2 allele (all $\mathrm{P}<0.05$ ). Multivariate logistic regression model showed that the $A L D H 2{ }^{*} 1 /{ }^{*} 1$ genotype was independently associated with CMBs $(\mathrm{P}=0.013)$, particularly for deep/mixed CMBs $(\mathrm{P}=0.008)$, and the association was more pronounced in men, non-drinkers or hypertension patients.

Conclusions: The results suggest that Han Chinese with $A L D H 2{ }^{*} 1 /{ }^{*} 1$ genotype may be more susceptible to CMBs than those with $A L D H 2$ *2 allele.

Keywords: Cerebral microbleeds (CMBs); aldehyde dehydrogenase 2 (ALDH2); genetic association study; brain magnetic resonance imaging (brain MRI)

Submitted Sep 25, 2019. Accepted for publication Dec 20, 2019.

doi: 10.21037/atm.2020.01.01

View this article at: http://dx.doi.org/10.21037/atm.2020.01.01 


\section{Introduction}

Stroke accounts for approximately $10 \%$ of all-cause deaths globally, and the estimated lifetime risk of stroke was about $25 \%$ after 25 years old $(1,2)$. Eastern Asia ranks first in incidence of stroke, particularly for China where stroke is the leading cause of death $(2,3)$. Fortunately, there exists a long subclinical phase of stroke that can be detected by neuroimaging, making it possible for intervention and prevention of the fatal disease.

Recently, cerebral small vessel disease (CSVD) has been identified as a stroke-related preclinical vascular disease in the brain $(4,5)$. Cerebral microbleeds (CMBs), a magnetic resonance imaging (MRI) marker of CSVD, have been found quite common in patients with stroke, as well as in general elderly population (5). Contrast to other CSVD markers such as lacune, white matter hyperintensity, and perivascular space, CMBs were observed to indicate both ischemic and hemorrhagic changes in the brain (4). Multiple epidemiological studies have demonstrated that CMBs are associated with increased risk of stroke (6), dementia (7) and mortality (8). So far, however, little is known about risk factors other than hypertension for CMBs, especially in general populations.

Considering the close association between heavy alcohol drinking and the risk of stroke (9), which can be modified by aldehyde dehydrogenase 2 ( $A L D H 2)$, a so-called alcohol flush gene (10), several studies have focused on the influence of variants at $A L D H 2$ on cerebrovascular disease (11-17). However, the results were inconsistent (11-17). $A L D H 2$ gene encodes the major enzyme in ethanol oxidation process (18), and the allelic variant *2 at the gene was observed to dramatically reduce enzyme activity. The $A L D H 2$ *2 allele is more prevalent in Asians than that in Caucasians (19), leading to less alcoholism in Asians (20) but the highest incidence of stroke. The unparallelism suggests that Asian may be more susceptible to stroke, particularly for those with $A L D H 2 * 1$ allele.

Herein, based on the population-based Taizhou Imaging Study (TIS), we conducted a cross-sectional study to evaluate the association between $A L D H 2$ gene polymorphisms and CMBs, the MRI marker for CSVD, the stroke-related preclinical vascular disease in general population in China. Our study may provide evidence for prevention of stroke in the high-risk population.

\section{Methods}

\section{Study design and participants}

The present study was conducted as a part of the TIS, an ongoing community population-based neuroimaging cohort, which originates from the Taizhou Longitudinal Study (21). The detailed description of TIS has been reported in our previous studies $(22,23)$. In brief, from March 2013 to January 2015, a total of 624 Han Chinese individuals aged 55-65 years without stroke, cancer, cardiovascular disease, psychiatric disorders, or other serious illness in two villages of Taixing were invited to participate the baseline survey of TIS, and received extensive physical, cognitive, and brain MRI examinations. Totally, 562 participants met the inclusion criteria were included in phase I of the TIS. Of these, 13 individuals without baseline whole blood samples were excluded and eventually 549 subjects were involved the present investigation. All related examinations were performed on the same day as the MRI examination at the Taizhou People's Hospital. The TIS was approved by the Ethics Committee of the School of Life Sciences, Fudan University, Shanghai, China (institutional review board approval number: 469), and all participants gave written informed consent.

\section{Brain MRI and CMBs assessment}

All the participants underwent brain MRI on the same 3.0T scanner (Magnetom Verio Tim scanner; Siemens, Erlangen, Germany) followed a pre-determined protocol. Two experienced neurologists (M.C. and Q.Y.) independently diagnosed CMBs according to the Standards for Reporting Vascular Changes on Neuroimaging (STRIVE) (24) through Horos software (version 1.1.7). In general, CMBs were defined as round or ovoid shaped local signal loss on T2*-weighted GRE sequence and with 2-5 $\mathrm{mm}$ diameter (no more than $10 \mathrm{~mm}$ ). The final CMBs diagnoses were based on their consensus and the final decision regarding to discrepancies were made by a senior neuroradiologist (W.J.T.). The kappa value of CMBs identification was 0.83. CMBs were divided into strictly lobar CMBs and deep/mixed CMBs by lesion location, as mixed CMBs shared similar characteristics with deep CMBs (25). The two typically CMBs location have their own pathologies distribution: strictly lobar CMBs usually located in frontal, 
Table 1 Characteristics of participants with and without CMBs

\begin{tabular}{|c|c|c|c|}
\hline \multirow[b]{2}{*}{ Characteristics } & \multirow{2}{*}{$\begin{array}{c}\text { Non-CMBs } \\
(n=446)\end{array}$} & \multicolumn{2}{|c|}{ CMBs location } \\
\hline & & $\begin{array}{c}\text { Deep/mixed } \\
\quad(n=55)\end{array}$ & Lobar $(n=48)$ \\
\hline Age, mean (SD), years & $59.02(2.72)$ & $59.71(2.57)$ & $60.12(2.56)^{*}$ \\
\hline Women, n (\%) & $233(52.2)$ & $33(60.0)$ & $33(68.8)$ \\
\hline BMI, mean (SD) & $24.13(3.32)$ & $24.45(3.96)$ & $23.45(2.16)$ \\
\hline Smoking ${ }^{\#}$ n (\%) & $162(36.7)$ & $18(33.3)$ & $11(22.9)$ \\
\hline Alcohol drinking ${ }^{\#}, \mathrm{n}(\%)$ & $133(30.3)$ & $15(27.8)$ & $11(22.9)$ \\
\hline Hypertension, n (\%) & $235(52.7)$ & $38(69.1)^{*}$ & $32(66.7)$ \\
\hline Diabetes, n (\%) & $61(13.7)$ & $9(16.4)$ & $3(6.3)$ \\
\hline Hyperlipidemia, n (\%) & $216(48.4)$ & $27(49.1)$ & $21(43.8)$ \\
\hline \multicolumn{4}{|l|}{$\begin{array}{l}\text { ALDH2 genotype } \\
(\mathrm{rs} 671)^{*}, \mathrm{n}(\%)\end{array}$} \\
\hline$* 1 / * 1$ & $249(55.8)$ & $42(76.4)$ & $31(64.6)$ \\
\hline${ }^{*} 1 /{ }^{*} 2$ & $173(38.8)$ & $10(18.2)$ & $16(33.3)$ \\
\hline${ }^{*} 2 /{ }^{*} 2$ & $24(5.4)$ & $3(5.5)$ & $1(2.1)$ \\
\hline
\end{tabular}

\#, 5 subjects had missing data for smoking and drinking status; *, $\mathrm{P}<0.05$ (compared with non-CMBs group). CMBs, cerebral microbleeds; SD, standard deviation; BMI, body mass index; $A L D H 2$, aldehyde dehydrogenase 2.

parietal, temporal, and occipital, whereas deep/mixed CMBs usually located in basal ganglia and thalamus, corpus callosum, and infratentorial including brain stem and cerebellum (25).

\section{DNA collection and genotyping}

Venous blood samples were drawn between 7:00 and 8:00 am after an overnight fasting by certified nurses in vacuum tubes with ethylenediaminetetraacetic acid (EDTA). DNA was extracted by using a whole-blood DNA extraction and purification kit (BaiO, Shanghai, China) according to the manufacturer's instructions. Genotyping was performed on coded genomic DNA samples using Axiom Precision Medicine Research Array (PMRA). All single nucleotide polymorphisms (SNPs) at $A L D H 2$ and the previous reported surrogate marker of $A L D H 2$ were extracted for preliminary analysis (Table S1). Results show that only rs2074356 and rs671 were associated with CMBs, however, rs2074356 is in high linkage disequilibrium with rs671 in our population $\left(\mathrm{R}^{2}=0.653\right)$. Therefore, the most well-known functional SNP rs671 (26), was selected as a surrogate
$A L D H 2$ genotyping marker for subsequent analyses. Since only a small number of individuals carry $A L D H 2 * 2 / * 2$ genotype $(\mathrm{n}=28,5.1 \%)$ in this study, we combined $A L D H 2$ ${ }^{*} 2 /{ }^{*} 2$ and ${ }^{*} 1 /{ }^{*} 2$ genotype as the reduced enzyme activity group, whereas participants with $A L D H 2{ }^{*} 1 /{ }^{*} 1$ genotype as the fully enzyme activity group (15). The SNP rs671 had a $100 \%$ call rate in all samples, and were in consistency with Hardy-Weinberg Equilibrium among CMB-free participants $(\mathrm{P}=0.374)$.

\section{Covariates}

In-person interview was performed to collect demographic characteristics and risk factors using a structured interviewer-administered questionnaire which included age, sex, habits of smoking and alcohol drinking. Alcohol drinking was defined as alcohol use at least once per week within recent 6 months. Calculation of body mass index (BMI) and definitions of hypertension, diabetes mellitus $(\mathrm{DM})$, and hyperlipidemia were described in our previous study (22).

\section{Statistical analysis}

The categorical variables were compared using Pearson's chi-squared test and were presented as frequencies (\%). Continuous variables were described as mean (standard deviation, SD) and were compared using Student's $t$-tests. Univariate and multivariate logistic regression models were used to estimate the odds ratios (ORs) and $95 \%$ confidence intervals (CIs) of CMBs with potential risk factors. Then we tested the multiplication interactions of $A L D H 2$ genotypes with sex, alcohol drinking and hypertension status in the risk of CMBs. Stratification analyses were then conducted by sex, alcohol drinking, and hypertension status, using *2 allele as the reference group. In multivariate models, model 1 was adjusted for age, sex, BMI, smoking, alcohol drinking while model 2 was additionally adjusted for hypertension, $\mathrm{DM}$, and hyperlipidemia. All analyses were performed using the $\mathrm{R}$ program (version 3.5.1, $\mathrm{R}$ core team), and two-tailed $\mathrm{P}<0.05$ was considered as statistical significant.

\section{Results}

The baseline characteristics for CMBs locations (absence, deep/mixed or lobar CMBs) of the 549 participants are presented in Table 1. In total, 103 individuals (18.8\%) were identified with CMBs, of whom 48 (46.6\%) were 
Table 2 Basic characteristics of participants across different $A L D H 2$ genotypes

\begin{tabular}{lrrr}
\hline Characteristics & ${ }^{*} 1 /{ }^{*} 1(\mathrm{n}=322)$ & $\begin{array}{c}{ }^{*} 1 /{ }^{*} 2 \text { and }{ }^{*} 2 /{ }^{*} 2 \\
(\mathrm{n}=227)\end{array}$ & P value \\
\hline Age, mean (SD), years & $59.36(2.66)$ & $58.94(2.77)$ & 0.075 \\
Women, n (\%) & $171(53.1)$ & $128(56.4)$ & 0.501 \\
BMI, mean (SD) & $24.30(3.43)$ & $23.82(3.12)$ & 0.098 \\
Smoking, $\mathrm{n}(\%)$ & $108(33.9)$ & $83(36.9)$ & 0.523 \\
Alcohol drinking ${ }^{\#}, \mathrm{n}(\%)$ & $121(37.8)$ & $38(16.9)$ & $<0.001$ \\
Hypertension, n (\%) & $191(59.3)$ & $114(50.2)$ & 0.043 \\
Diabetes, n (\%) & $41(12.7)$ & $32(14.1)$ & 0.737 \\
Hyperlipidemia, n (\%) & $157(48.8)$ & $107(47.1)$ & 0.774 \\
CMBs, n (\%) & $73(22.7)$ & $30(13.2)$ & 0.007 \\
Deep/mixed & $42(13.0)$ & $13(5.7)$ & 0.008 \\
Lobar & $31(9.6)$ & $17(7.5)$ & 0.471 \\
\hline
\end{tabular}

", 5 subjects had missing data for smoking and drinking status. $A L D H 2$, aldehyde dehydrogenase 2; SD, standard deviation; $\mathrm{BMI}$, body mass index; CMBs, cerebral microbleeds.

lobar CMBs and 55 (53.4\%) were deep/mixed CMBs. The participants with $\mathrm{CMBs}$ was slightly older than those without, with mean age of 59.90 vs. 59.02 years $(\mathrm{P}=0.003)$. The CMBs patients were more likely to be women $(64.1 \%$ vs. $52.2 \% ; \mathrm{P}=0.030)$, have hypertension $(68.0 \%$ vs. $52.7 \%$; $\mathrm{P}=0.005)$, and carry $A L D H 2$ *2 allele $(\mathrm{P}=0.020)$ than nonCMBs. No significant difference was observed for BMI, cigarette smoking, DM and hyperlipidemia between CMBs and non-CMBs.

We classified all subjects into two groups by genotype of $A L D H 2$, i.e., $A L D H 2{ }^{*} 1 /{ }^{*} 1$ (*1 allele group) vs. $A L D H 2$ ${ }^{*} 1 /{ }^{*} 2$ and ${ }^{*} 2 /{ }^{*} 2$ (*2 allele group). As shown in Table 2, alcohol consumption was less frequent in $A L D H 2$ *2 allele group $(\mathrm{P}<0.001)$, whereas hypertension $(\mathrm{P}=0.043)$ and $\mathrm{CMBs}(\mathrm{P}=0.007)$, particularly deep/mixed $\mathrm{CMBs}$ $(\mathrm{P}=0.008)$, were more common in $A L D H 2$ * 1 allele group. No significant differences were found for other vascular risk factors such as age, sex, BMI, smoking, DM, and hyperlipidemia.

Table 3 shows the association between $A L D H 2$ gene polymorphisms and CMBs. $A L D H 2{ }^{*} 1 /{ }^{*} 1$ genotype was significantly associated with CMBs before (OR: 1.93, 95\% CI: 1.21-3.06) and after adjusting for other vascular risk factors (OR: 1.87, 95\% CI: 1.14-3.06). The association was more pronounce in deep/mixed CMBs (OR: 2.53,
95\% CI: 1.28-5.03) than that in lobar CMBs (OR: 1.39, 95\% CI: 0.72-2.70). Further analysis observed a significant interaction between $A L D H 2$ genotype and sex or hypertension on $\mathrm{CMBs}$ (all $\mathrm{P}<0.05$ ). $A L D H 2$ genotype was also observed to have a borderline interaction with alcohol drinking $(\mathrm{P}=0.076)$ on $\mathrm{CMBs}$ after adjusting possible confounders. Stratified analyses by sex, alcohol drinking and hypertension status showed that $A L D H 2{ }^{*} 1 /{ }^{*} 1$ genotype was significantly associated with CMBs only among men (OR: 4.51, 95\% CI: 1.70-11.97), non-alcohol drinkers (OR: $1.76,95 \%$ CI: $1.02-3.04 ; \mathrm{P}=0.044)$ or hypertension patients (OR: 2.21, 95\% CI: 1.17-4.17).

\section{Discussion}

The present study aimed to investigate the relationship between the polymorphisms of $A L D H 2$ gene and CMBs. Based on the baseline data of TIS (phase I), we found that $A L D H 2{ }^{*} 1{ }^{*} 1$ genotype was associated with great odds of CMBs in aged 55-65 years Han Chinese population, especially in men, non-drinkers or hypertension patients.

As a member of detoxifying enzymes $A L D H$ family, $A L D H 2$ plays an important role in the process of metabolizing acetaldehyde to acetic acid (27). $A L D H 2 * 2$ allele is the most common single point mutation related with reduce enzyme activity. The mutation is more prevalent in Asian population (28). Similar to this previous report, we found $41.4 \%$ participants of this study with $A L D H 2 * 2$ allele and $5.1 \%$ individuals were $* 2 / 2$ genotype. We also found that individuals with $A L D H 2$ *2/*2 genotype were less likely to drink alcohol than those with * $1 /{ }^{*} 1$ genotype.

$A L D H 2 * 2$ allele showed strong protection against stroke in Han Chinese with a history of heavy drinking (14), and excessive ethanol consumption aggravates the ischemic brain injury by inhibiting $A L D H 2$ gene function (29). Moreover, $A L D H 2$ gene could clear 4-hydroxy-2-nonenal and down regulate aquaporin 4 to protect against stroke $(30,31)$. Recently, several studies found that $A L D H 2 * 2$ allele acted as an independent risk factor for ischemic stroke and cerebral infarctions in Chinese (11-13). However, many of other studies reported that the * 1 allele of $A L D H 2$ appears to be a significant risk factor for intracranial vascular stenosis in ischemic stroke (16), multiple lacunar infarcts $(15)$, and stroke $(14,17)$ in Asian population, which were similar with our results. We found that $A L D H 2{ }^{*} 1{ }^{*} 1$ genotype was positively associated with CMBs in Chinese elderly population. So far, there is no consistent conclusion 
Table 3 Stratified analysis for $A L D H 2$ as risk factor in participants with CMBs by location, sex, drinking and hypertension status

\begin{tabular}{|c|c|c|c|c|c|c|}
\hline ALDH2 (*1 vs. *2) & \multicolumn{2}{|c|}{ Univariate model } & \multicolumn{2}{|l|}{ Model 1} & \multicolumn{2}{|c|}{ Model 2} \\
\hline Overall & $1.93(1.21-3.06)$ & 0.006 & $1.91(1.17-3.12)$ & 0.009 & $1.87(1.14-3.06)$ & 0.013 \\
\hline \multicolumn{7}{|l|}{ CMBs location } \\
\hline Deep/mixed & $2.56(1.34-4.89)$ & 0.005 & $2.55(1.29-5.05)$ & 0.007 & $2.53(1.28-5.03)$ & 0.008 \\
\hline \multicolumn{7}{|l|}{ Sex } \\
\hline Men & $3.26(1.37-7.75)$ & 0.008 & $4.43(1.70-11.49)$ & 0.002 & $4.51(1.70-11.97)$ & 0.003 \\
\hline Women & $1.53(0.87-2.71)$ & 0.140 & $1.29(0.71-2.34)$ & 0.401 & $1.28(0.70-2.34)$ & 0.429 \\
\hline \multicolumn{7}{|l|}{ Alcohol consumption } \\
\hline \multicolumn{7}{|l|}{ Hypertension status } \\
\hline Hypertension & $2.19(1.20-4.01)$ & 0.011 & $2.21(1.18-4.15)$ & 0.014 & $2.21(1.17-4.17)$ & 0.014 \\
\hline Non-hypertension & $1.39(0.66-2.93)$ & 0.393 & $1.51(0.67-3.39)$ & 0.323 & $1.52(0.67-3.43)$ & 0.318 \\
\hline
\end{tabular}

Model 1 was adjusted for sex, age, BMI, smoking, and drinking; model 2 was additionally adjusted for hypertension, DM, and hyperlipidemia. $A L D H 2$, aldehyde dehydrogenase 2; CMBs, cerebral microbleeds; OR, odds ratio; Cl, confidence interval; BMI, body mass index; DM, diabetes mellitus.

about the association between polymorphisms of $A L D H 2$ and stroke. Additionally, we did not find certain reports concentrating on the relationship between the $A L D H 2$ and CMBs in other population.

Hypertension has been proven to be a definite risk factor for CMBs $(4,5)$. As a multifactorial disease, the underlying pathogenesis of CMBs points to arteriolosclerosis and cerebral amyloid angiopathy, and is strongly associated with aging and hypertension (4). Previous studies found that deep/mixed CMBs were associated with hypertensive vasculopathy, while strictly lobar CMBs were related to cerebral amyloid angiopathy (4). We found a higher prevalence of hypertension in participants with $A L D H 2$ * $1 /{ }^{*} 1$ genotype than that in $A L D H 2{ }^{*} 2$ allele carriers (Table 2). Similarly, Wu et al. found that $A L D H 2{ }^{*} 1{ }^{*} 1$ genotype was significantly associated with essential hypertension, especially in men in a case-control study in China and a meta-analysis Asia (32). Furthermore, a metaanalysis of genome-wide association studies found that variants in $A L D H 2$ were associated with blood pressure in east Asians (33). In present study, we found that $A L D H 2$ ${ }^{*} 1 /{ }^{*} 1$ genotype is associated with greater odds of deep/ mixed CMBs but not for lobar CMBs, which consistent with the CMBs location pathologies distribution. In addition, $A L D H 2$ * 1 allele was reported associated with intracranial vascular stenosis (16), atherosclerosis (34), and higher plasma homocysteine level (16) and so on, which are all the risk factors for cerebrovascular disease.

We found that the association between $A L D H 2$ gene polymorphisms and CMBs was more pronounced in men, which is consistent with several previous studies. Nagasawa et al. (15) reported that $A L D H 2{ }^{*} 1{ }^{*} 1$ genotype was significantly associated with multiple lacunar infarcts in Japanese men. $A L D H 2 * 2$ variant allele was shown as an independent protective factor against high alcohol consumption in male stroke patients in China (14). Cheng et al. (35) found $A L D H 2$ gene polymorphisms was a risk factor in modified ischemic stroke in Chinese men, but not in women. It is worth noting that $A L D H 2{ }^{*} 1 /{ }^{*} 1$ was significantly associated with essential hypertension in men (32), which may be a potential mechanism for high risk of CMBs and stroke in men with $A L D H 2{ }^{*} 1 /{ }^{*} 1$ genotype. Whether alcohol drinking is an independent risk factor for stroke is controversial (36). Heavy alcohol consumption can predict incident CMBs in deep region but not in lobar brain regions (37), and individuals with $A L D H 2{ }^{*} 1 /{ }^{*} 1$ genotype tend to be alcohol 
drinker, which might increase the risk for hypertension (38). However, we did not find significant relationship between alcohol drinking and CMBs in this study. We conducted stratified analysis and found that $A L D H 2{ }^{*} 1 /{ }^{*} 1$ genotype was a risk factor for CMBs, especially among non-drinkers. In addition, we did not find significant association between alcohol drinking and hypertension in our participants. To some extent, these results suggest that $A L D H 2{ }^{*} 1 /{ }^{*} 1$ genotype leading to hypertension might not entirely result from alcohol consumption. By stratification of the blood pressure, it showed that participants with $A L D H 2{ }^{*} 1 /{ }^{*} 1$ genotype had an additive effect on CMBs in hypertensive patients. Therefore, the definite biological mechanisms underlying this relationship need further to investigate.

To our best knowledge, this is the first study to demonstrate that a polymorphism at $A L D H 2$ was associated with CMBs in elderly population in China. However, several limitations should be acknowledged. First, selection bias could not be avoided in the present study, since our subjects were not selected randomly, but recruited on volunteering in rural China. Second, the sample size was relatively small, limiting statistical power to identify modifying effects of lower exposures in association of $A L D H 2$ polymorphisms with CMBs. Additionally, evaluation of the association between $A L D H 2$ polymorphisms and progression of CMBs (i.e., increase of CMBs number) would be informative in future perspective studies. Finally, we didn't collect information on amount of alcohol drinking, and could not evaluate the possible different effects of low, medium or heavy alcohol drinking on CMBs.

\section{Conclusions}

In summary, the present study is the first to demonstrate that the $A L D H 2{ }^{*} 1 /{ }^{*} 1$ genotype is positively associated with CMBs in rural Chinese population aged 55-65 years, particularly among men, non-alcohol drinkers or hypertension patients. A multi-center large scale prospective study is warranted to confirm our findings in the future.

\section{Acknowledgments}

We would like to thank Dr. Weijun Tang, Qi Yang and He Wang for their help with MRI image rating and neuropsychological data reviewing. We are grateful to all the participants for their cooperation. We thank the personnel from Fudan University Taizhou Institute of Health Sciences, the staff of Taizhou People's Hospital and
Taixing Disease Control and Prevention Center (CDC) for their assistance in conducting the medical interviews and data collection.

Funding: This study was supported by the National Key Research and Development program of China (grant number: 2017YFC0907000, 2017YFC0907500, 2017YFC0907200), the International Science and Technology Cooperation Program of China (grant number: 2014DFA32830), the Key Research and Development Plans of Jiangsu Province, China (grant number: BE2016726), Shanghai Municipal Science and Technology Major Project (grant number: 2017SHZDZX01), the Key Basic Research Grants from Science and Technology Commission of Shanghai Municipality, China (grant number: 16JC1400501), and the Key Technology Research and Development Program of Taizhou (grant number: TS201833).

\section{Footnote}

Conflicts of Interest: The authors have no conflicts of interest to declare.

Ethical Statement: The authors are accountable for all aspects of the work in ensuring that questions related to the accuracy or integrity of any part of the work are appropriately investigated and resolved. The study was approved by the Ethics Committee of the School of Life Sciences, Fudan University, Shanghai, China (institutional review board approval number: 469), and all participants gave written informed consent.

Open Access Statement: This is an Open Access article distributed in accordance with the Creative Commons Attribution-NonCommercial-NoDerivs 4.0 International License (CC BY-NC-ND 4.0), which permits the noncommercial replication and distribution of the article with the strict proviso that no changes or edits are made and the original work is properly cited (including links to both the formal publication through the relevant DOI and the license). See: https://creativecommons.org/licenses/by-nc-nd/4.0/.

\section{References}

1. GBD 2016 Causes of Death Collaborators. Global, regional, and national age-sex specific mortality for 264 causes of death, 1980-2016: a systematic analysis for the Global Burden of Disease Study 2016. Lancet 
2017;390:1151-210.

2. GBD 2016 Lifetime Risk of Stroke Collaborators, Feigin VL, Nguyen G, et al. Global, regional, and countryspecific lifetime risks of stroke, 1990 and 2016. N Engl J Med 2018;379:2429-37.

3. Yang G, Wang Y, Zeng Y, et al. Rapid health transition in China, 1990-2010: findings from the Global Burden of Disease Study 2010. Lancet 2013;381:1987-2015.

4. Pantoni L. Cerebral small vessel disease: from pathogenesis and clinical characteristics to therapeutic challenges. Lancet Neurol 2010;9:689-701.

5. Smith EE, Saposnik G, Biessels GJ, et al. Prevention of stroke in patients with silent cerebrovascular disease: a scientific statement for healthcare professionals from the American Heart Association/American Stroke Association. Stroke 2017;48:e44-71.

6. Akoudad S, Portegies ML, Koudstaal PJ, et al. Cerebral microbleeds are associated with an increased risk of stroke: the Rotterdam study. Circulation 2015;132:509-16.

7. Akoudad S, Wolters FJ, Viswanathan A, et al. Association of cerebral microbleeds with cognitive decline and dementia. JAMA Neurol 2016;73:934-43.

8. Akoudad S, Ikram MA, Koudstaal PJ, et al. Cerebral microbleeds and the risk of mortality in the general population. Eur J Epidemiol 2013;28:815-21.

9. Zhang C, Qin YY, Chen Q, et al. Alcohol intake and risk of stroke: a dose-response meta-analysis of prospective studies. Int J Cardiol 2014;174:669-77.

10. Chen CC, Lu RB, Chen YC, et al. Interaction between the functional polymorphisms of the alcohol-metabolism genes in protection against alcoholism. Am J Hum Genet 1999;65:795-807.

11. Sung YF, Lu CC, Lee JT, et al. Homozygous ALDH2*2 is an independent risk factor for ischemic stroke in Taiwanese men. Stroke 2016;47:2174-9.

12. Sun S, He J, Zhang Y, et al. Genetic polymorphisms in the ALDH2 gene and the risk of ischemic stroke in a Chinese Han population. Oncotarget 2017;8:101936-43.

13. Li QY, Zhao NM, Ma JJ, et al. ALDH2*2 allele is a negative risk factor for cerebral infarction in Chinese women. Biochem Genet 2015;53:260-7.

14. Yao CT, Cheng CA, Wang HK, et al. The role of ALDH2 and $\mathrm{ADH} 1 \mathrm{~B}$ polymorphism in alcohol consumption and stroke in Han Chinese. Hum Genomics 2011;5:569-76.

15. Nagasawa $H$, Wada $M$, Arawaka $S$, et al. A polymorphism of the aldehyde dehydrogenase 2 gene is a risk factor for multiple lacunar infarcts in Japanese men: the Takahata Study. Eur J Neurol 2007;14:428-34.
16. Qu Y, Zhang HL, Yu LM, et al. Aldehyde dehydrogenase 2 polymorphism as a protective factor for intracranial vascular stenosis in ischemic stroke in Han Chinese. Int J Neurosci 2016;126:342-7.

17. Shin C, Kwack K, Cho NH, et al. Sex-specific differences in the association of a common aldehyde dehydrogenase 2 gene polymorphism and alcohol consumption with stroke risk in a Korean population: a prospective cohort study. Nutr Res Pract 2015;9:79-86.

18. Chen YC, Peng GS, Wang MF, et al. Polymorphism of ethanol-metabolism genes and alcoholism: correlation of allelic variations with the pharmacokinetic and pharmacodynamic consequences. Chem Biol Interact 2009;178:2-7.

19. Goedde HW, Harada S, Agarwal DP. Racial differences in alcohol sensitivity: a new hypothesis. Hum Genet 1979;51:331-4.

20. Matsushita S, Higuchi S. Review: use of Asian samples in genetic research of alcohol use disorders: genetic variation of alcohol metabolizing enzymes and the effects of acetaldehyde. Am J Addict 2017;26:469-76.

21. Wang X, Lu M, Qian J, et al. Rationales, design and recruitment of the Taizhou Longitudinal Study. BMC Public Health 2009;9:223.

22. Jiang Y, Fan Z, Wang Y, et al. Low bone mineral density is not associated with subclinical atherosclerosis: a population-based study in rural China. Cardiology 2018;141:78-87.

23. Li S, Fang F, Cui M, et al. Incidental findings on brain MRI among Chinese at the age of 55-65 years: the Taizhou Imaging Study. Sci Rep 2019;9:464.

24. Wardlaw JM, Smith EE, Biessels GJ, et al. Neuroimaging standards for research into small vessel disease and its contribution to ageing and neurodegeneration. Lancet Neurology 2013;12:822-38.

25. Ding J, Sigurdsson S, Garcia M, et al. Risk factors associated with incident cerebral microbleeds according to location in older people the age, gene/environment susceptibility (AGES)-Reykjavik study. JAMA Neurol 2015;72:682-8.

26. Suo C, Yang Y, Yuan Z, et al. Alcohol intake interacts with functional genetic polymorphisms of aldehyde dehydrogenase (ALDH2) and alcohol dehydrogenase $(\mathrm{ADH})$ to increase esophageal squamous cell cancer risk. J Thorac Oncol 2019;14:712-25.

27. Qu Y, Zhang H, Li H, et al. Aldehyde dehydrogenase 2 (ALDH2) Glu504Lys polymorphism affects collateral circulation and short-term prognosis of acute cerebral 
infarction patients. Med Sci Monit 2017;23:4559-66.

28. Eng MY, Luczak SE, Wall TL. ALDH2, ADH1B, and ADH1C genotypes in Asians: a literature review. Alcohol Res Health 2007;30:22-7.

29. Wang W, Lin LL, Guo JM, et al. Heavy ethanol consumption aggravates the ischemic cerebral injury by inhibiting ALDH2. Int J Stroke 2015;10:1261-9.

30. Guo JM, Liu AJ, Zang P, et al. ALDH2 protects against stroke by clearing 4-HNE. Cell Res 2013;23:915-30.

31. Li Y, Liu SL, Qi SH. ALDH2 protects against ischemic stroke in rats by facilitating 4-HNE clearance and AQP4 down-regulation. Neurochem Res 2018;43:1339-47.

32. Wu Y, Ni J, Cai X, et al. Positive association between ALDH2 rs671 polymorphism and essential hypertension: a case-control study and meta-analysis. PLoS One 2017;12:e0177023.

33. Kato N, Takeuchi F, Tabara Y, et al. Meta-analysis of genome-wide association studies identifies common variants associated with blood pressure variation in east

Cite this article as: Zhu Z, Jiang Y, Cui M, Wang Y, Li S, Xu K, Zhang K, Zhu C, Xu W, Jin L, Ye W, Suo C, Chen X. ALDH2 rs671 polymorphisms and the risk of cerebral microbleeds in Chinese elderly: the Taizhou Imaging Study. Ann Transl Med 2020;8(5):229. doi: 10.21037/atm.2020.01.01
Asians. Nat Genet 2011;43:531-8.

34. Zhong S, Li L, Zhang YL, et al. Acetaldehyde dehydrogenase 2 interactions with LDLR and AMPK regulate foam cell formation. J Clin Invest 2019;129:252-67.

35. Cheng X, Xu J, Gu M, et al. Genetic variants in ALDH2 predict risk of ischemic stroke in a Chinese population. Gene 2018;678:49-54.

36. Reynolds K, Lewis B, Nolen JD, et al. Alcohol consumption and risk of stroke: a meta-analysis. JAMA 2003;289:579-88.

37. Caunca MR, Del Brutto V, Gardener H, et al. Cerebral microbleeds, vascular risk factors, and magnetic resonance imaging markers: The Northern Manhattan Study. J Am Heart Assoc 2016. doi: 10.1161/JAHA.116.003477.

38. Tsuchihashi-Makaya M, Serizawa M, Yanai K, et al. Geneenvironmental interaction regarding alcohol-metabolizing enzymes in the Japanese general population. Hypertens Res 2009;32:207-13. 
Supplementary

Table S1 Analysis for ALDH2 as risk factor in participants with CMBs

\begin{tabular}{|c|c|c|c|c|c|c|}
\hline ALDH2 (*1 vs. *2) & \multicolumn{2}{|c|}{ Univariate model } & \multicolumn{2}{|c|}{ Model 1} & \multicolumn{2}{|c|}{ Model 2} \\
\hline rs671 & $1.93(1.21-3.06)$ & 0.006 & $1.91(1.17-3.12)$ & 0.009 & $1.87(1.14-3.06)$ & 0.013 \\
\hline rs2074356 & $2.13(1.25-3.64)$ & 0.004 & $2.14(1.23,3.72)$ & 0.005 & $2.09(1.20-3.65)$ & 0.007 \\
\hline rs2238151 & $1.31(0.57-3.03)$ & 0.521 & $1.23(0.53,2.87)$ & 0.628 & $1.31(0.53-3.10)$ & 0.532 \\
\hline
\end{tabular}

Model 1 was adjusted for sex, age, BMI, smoking, and drinking; model 2 was additionally adjusted for hypertension, DM, and hyperlipidemia. $A L D H 2$, aldehyde dehydrogenase 2; CMBs, cerebral microbleeds; OR, odds ratio; Cl, confidence interval; BMI, body mass index; DM, diabetes mellitus. 